\title{
Renormalization of spin excitations and Kondo effect in open-shell nanographenes
}

\author{
David Jacob $\odot,{ }^{1,2, *}$ Ricardo Ortiz, ${ }^{3,4}$ and Joaquín Fernández-Rossier ${ }^{5, \dagger}$ \\ ${ }^{1}$ Departamento de Polímeros y Materiales Avanzados: Física, Química y Tecnología, \\ Universidad del País Vasco UPV/EHU, Av. Tolosa 72, E-20018 San Sebastián, Spain \\ ${ }^{2}$ IKERBASQUE, Basque Foundation for Science, Plaza Euskadi 5, E-48009 Bilbao, Spain \\ ${ }^{3}$ Departamento de Física Aplicada, Universidad de Alicante, E-03690 San Vicente del Raspeig, Spain \\ ${ }^{4}$ Departamento de Química Física, Universidad de Alicante, E-03690 San Vicente del Raspeig, Spain \\ ${ }^{5}$ QuantaLab, International Iberian Nanotechnology Laboratory (INL), 4715-330 Braga, Portugal
}

(Received 6 April 2021; revised 19 July 2021; accepted 20 July 2021; published 3 August 2021)

\begin{abstract}
We study spin excitations and the Kondo effect in open-shell nanographenes, motivated by recent scanning tunneling inelastic spectroscopy experiments. Specifically, we consider three systems: the triangulene, the extended triangulene with rocket shape, both with an $S=1$ ground state, and a triangulene dimer with $S=0$ on account of intermolecular exchange. We focus on the consequences of hybridization of the nanographene zero modes with a conducting substrate on the $d I / d V$ line shapes associated with spin excitations. The partially filled nanographene zero modes coupled to the conduction electrons in the substrate constitute multiorbital Anderson impurity models that we solve in the one-crossing approximation, which treats the coupling to the substrate to infinite order. We find that the coupling to the substrate leads to (i) renormalization of the spin flip excitation energies of the bare molecule, (ii) broadening of the spectral features, and (iii) the emergence of zero bias Kondo peaks for the $S=1$ ground states. The calculated substrate induced shift of the spin excitation energies is found to be significantly larger than their broadening, which implies that this effect has to be considered when comparing experimental results and theory.
\end{abstract}

DOI: 10.1103/PhysRevB.104.075404

\section{INTRODUCTION}

Open-shell nanographenes (NGs) have been studied theoretically for many decades [1-8] due to their very peculiar magnetic properties, with local moments associated with $\pi$ molecular states, very small magnetic anisotropy, and strong quantum spin fluctuations. However, their large chemical reactivity was a great obstacle for their experimental study. This situation has changed dramatically with the advent of on-surface synthesis techniques [9-11] combined with surface scanning probe techniques, such as atomic force and scanning tunneling microscopes (AFM and STM). These techniques make it possible now to synthesize an increasing number of open-shell NGs, such as ribbons with zigzag edges [12], the Clar's goblet [13], rhombenes [14], triangulenes [15-18], heptauthrene [19], and others [20-25], and to probe their electronic properties with atomic scale resolution.

A prominent challenge in this new research area is to probe the spin properties of open-shell NGs [26]. STM enables us to carry out inelastic electron tunnel spectroscopy (IETS) with atomic resolution (for a review of this topic, see, e.g., Ref. [27]). Two types of features signal the presence of open-shell configurations: zero-bias Kondo peaks and the observation of steplike features in the $d I / d V$ spec-

\footnotetext{
*david.jacob@ehu.es

$\dagger$ On leave from Departamento de Física Aplicada, Universidad de Alicante, E-03690 San Vicente del Raspeig, Spain.
}

tra at bias voltages $V= \pm V_{\text {ex }}$. However, whereas inelastic steps indicate the existence of an excitation at energy $e V_{\mathrm{ex}}$, their spin-dependent origin can only be confirmed directly if application of a magnetic field shifts the energy of that excitation. This approach has been thoroughly used in the past two decades to probe spin excitations of individual magnetic adatoms [28-32], single magnetic molecules [33] and adatom chains [34-36].

The direct experimental confirmation of the spin nature of the excitations cannot be carried out when the Zeeman shift of the excitation energy, in the range of $g \mu_{B} \simeq 0.12 \mathrm{meV}$ per $\mathrm{T}$, is smaller than the spectral resolution of IETS, controlled by temperature and broadening due to the lifetime of the spin excitations. The latter is governed by the strength of the effective exchange interactions to the substrate. In the case of NGs deposited directly on gold [13,14,17,20-22], broadening is definitely larger than Zeeman splitting, making it necessary to rely on the comparison with theory. The situation may be different in the case of the Kondo effect in $S=1$ NGs where the narrow Kondo peak splits when the Zeeman energy exceeds the Kondo temperature. Moreover, as reported recently by $\mathrm{Li}$ and co-workers [21], in the case of an underscreened Kondo effect, due to the residual magnetic moment of the NG, the Zeeman splitting of the Kondo resonance can already be observed at relatively small magnetic fields, limited only by thermal broadening.

Direct comparison between the excitation energies obtained from high-level quantum chemistry calculations and the singlet-triplet excitation energies yields fairly good 
agreement with the experimental observations in bowties $[8,13]$ and rhombenes [14]. However, it is known that the coupling of a magnetic system to the conduction electrons of the substrate not only broadens the spectral function of the spin excitations, but it also produces a renormalization of the energies, i.e., a shift of the step position [37-41], which has to be taken into account when comparing theory and experiment. In the case of a (spin) degenerate ground state (GS), the coupling to the substrate gives rise to the Kondo effect, screening the magnetic moment of the adsorbate [42]. This certainly occurs in NGs whose GS has $S=1 / 2$, but it has also been observed in NGs predicted to have $S=1[19,21]$. Note that the NG hosts the localized electrons, while the conduction electrons are in the substrate. This is different from the case of the Kondo effect of magnetic impurities and point defects in graphene [43-49].

In this work, we address all three effects, i.e., broadening and shift of IETS steps, and the emergence of zero-bias peaks, in the same theoretical framework. To the best of our knowledge, previous theory work has not addressed these in the context of nanographenes. We model the NGs using the Hubbard Hamiltonian solved by means of exact numerical diagonalization in the subspace defined by the zero modes (ZMs) of the NG. The energies obtained for this model compare well with higher level quantum chemistry calculations $[8,14]$. The NG coupled to the substrate is then described in terms of an Anderson impurity model (AIM). Here we make no attempt to describe the substrate from first principles. Instead, we use the strength of the coupling to the substrate, assumed to be the same for all atoms in the $\mathrm{NG}$, as a tunable parameter in the calculation. The AIM is solved in the one-crossing approximation (OCA), which consists in a diagrammatic expansion in the coupling strength to infinite order [50]. This yields the spectral function of the ZMs of the NG flake, directly related to the $d I / d V$ in the tunneling regime $[41,51]$.

We choose three NG systems that have been studied experimentally, shown in Fig. 1: (i) antiferromagnetically coupled dimers [17] of $S=1$ triangulenes with an $S=0$ GS, (ii) $S=1$ graphene triangulene [15], and (iii) $S=1$ extended triangulene [21], referred to as a "rocket" structure from now on.

\section{MODEL AND METHOD}

We consider the following Hamiltonian to describe a NG flake on a metallic substrate:

$$
\mathcal{H}=\mathcal{H}_{\text {ng }}+\mathcal{H}_{\text {sub }}+\mathcal{V}_{\text {hyb }} .
$$

The first term in (1) describes the NG flake in terms of a Hubbard model [8]:

$$
\mathcal{H}_{\mathrm{ng}}=\sum_{\substack{\langle i, j\rangle \\ \sigma}} t\left(c_{i \sigma}^{\dagger} c_{j \sigma}+c_{j \sigma}^{\dagger} c_{i \sigma}\right)+\sum_{i}\left(\epsilon_{0} n_{i}+U n_{i \uparrow} n_{i \downarrow}\right),
$$

where $c_{i \sigma}^{\dagger}\left(c_{j \sigma}\right)$ create (destroy) an electron of spin $\sigma \in\{\uparrow, \downarrow\}$ at carbon site $i(j)$ of the NG, $n_{i \sigma}=c_{i \sigma}^{\dagger} c_{i \sigma}$, and $n_{i}=\sum_{\sigma} n_{i \sigma}$. The model is determined by the first-neighbor hopping $t$ (second- and third-neighbor hoppings are neglected here), the atomic Hubbard $U$, and the carbon on-site energy $\epsilon_{0}$. We (a)

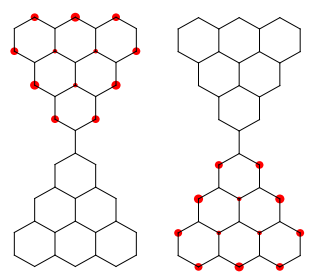

(c)

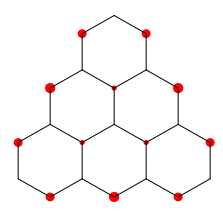

(e)

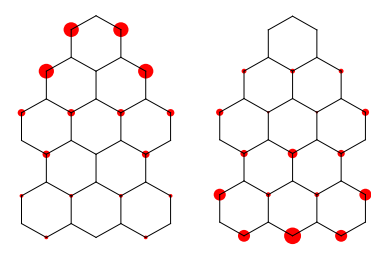

(b)

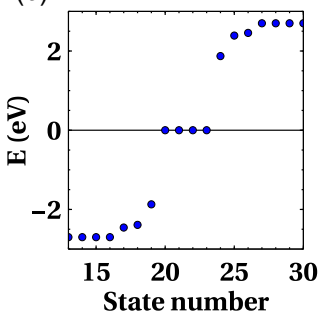

(d)

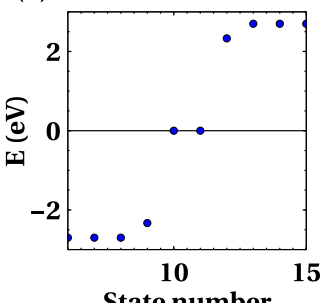

(f)

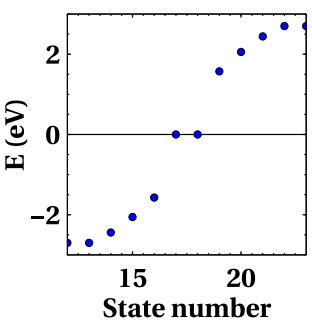

FIG. 1. Atomic structures with graphical representations of the ZMs [(a),(c),(e)] and corresponding single-particle spectra [(b),(d),(f)] for $t=-2.7 \mathrm{eV}$ of the three NGs discussed in the paper: triangulene dimer [(a),(b)], single triangulene [(c),(d)], and "rocket" structure [(e),(f)]. The size of the red circles in the structures (a), (c), and (e) yields the weight $\left|\psi_{\alpha}(i)\right|^{2}$ of the $E=0$ wave function $\psi_{\alpha}$ on carbon site $i$. Note that the other two ZMs in the case of the dimer and the other ZM for the single triangulene have the same weights (but different phases) as the ones already depicted in (a) and (c).

assume $t=-2.7 \mathrm{eV}$ and take $U$ as an adjustable parameter in the range of $U \simeq 1.5|t| \simeq 4 \mathrm{eV}$. First-principles estimates yield $U \simeq 9 \mathrm{eV}$ for graphene [52]. The fact that smaller values provide a better agreement with the experiments is probably due to screening of the Coulomb interaction by the conducting substrate.

The second term in (1) describes the conduction electron bath in the substrate, $\mathcal{H}_{\text {sub }}=\sum_{k, \sigma} \epsilon_{k} b_{k \sigma}^{\dagger} b_{k \sigma}$, where $b_{k \sigma}\left(b_{k \sigma}^{\dagger}\right)$ destroys (creates) an electron in bath state $k$ with spin $\sigma$. Without loss of generality we set the chemical potential of the conduction electron bath to zero $(\mu=0)$. The third term in (1) is the hybridization between the NG states and the conduction electron bath, $\mathcal{V}_{\text {hyb }}=\sum_{k, \sigma, i} V_{k, i} b_{k \sigma}^{\dagger} c_{i \sigma}+$ H.c. Integrating out the conduction electron degrees of freedom, we obtain the hybridization function, $\Delta_{i}(\omega)=\sum_{k}\left|V_{k, i}\right|^{2} /\left(\omega^{+}-\epsilon_{k}\right)$, which describes shift (real part) and broadening (imaginary part) of carbon site $i$ due to the coupling to the bath; as usual, $\omega^{+}=$ $\omega+i \eta$ shifting the poles/branch cut of the bath Green's function $1 /\left(\omega-\epsilon_{k}\right)$ from the real axis infinitesimally to the lower complex plain in order to make $\Delta_{i}(\omega)$ analytical in the upper complex plain. Here we assume the wide-band limit, i.e., the single-particle broadening $\Gamma_{i} \equiv-\operatorname{Im} \Delta_{i}(\omega)$ is assumed to 
be constant within a large energy window, and thus the real part of the hybridization function vanishes, $\operatorname{Re} \Delta_{i}(\omega)=0$. Moreover, we assume that each carbon site couples equally to the substrate, i.e., $\Gamma_{i}=\Gamma=$ const. These are reasonable approximations for noble metal substrates in the low-energy (or low-bias) regime in which we are interested.

As the many-body Hilbert space grows exponentially with the number of single-particle orbitals (or sites), it is not possible to numerically diagonalize (2) for the entire NG. One possibility is to treat the NG in the dynamical mean-field approximation (DMFA) adapted to nanoscale systems $[53,54]$. However, DMFA neglects nonlocal correlations, which are known to be important in low-dimensional systems. Instead we focus on a small subspace $\mathrm{C}$ of the full many-body Hilbert space defined by a subset of the molecular orbitals $\left\{\psi_{\alpha}\right\}$ of the NG. Specifically, we choose the so-called ZMs, which are the zero-energy eigenstates of the hopping part of (2) which host the unpaired electrons, responsible for the magnetism of open-shell NGs $[4,5,8,26]$. The ZMs can be expanded in the site basis $\{|i\rangle\}$ as $\left|\psi_{\alpha}\right\rangle=\sum_{i} \psi_{\alpha}(i)|i\rangle$. Figure 1 shows graphical representations of the ZMs for the three NG systems considered here.

The subspace $\mathrm{C}$ coupled to the substrate defines a multiorbital AIM. The impurity shell is given by the projection of the NG Hamiltonian (2) onto $\mathrm{C}$ and takes the form

$$
\mathcal{H}_{\mathrm{C}}=\sum_{\alpha} \varepsilon_{\mathrm{C}} N_{\alpha}+\sum_{\substack{\alpha, \alpha^{\prime}, \beta, \beta^{\prime} \\ \sigma, \sigma^{\prime}}} \tilde{U}_{\alpha \alpha^{\prime} \beta \beta^{\prime}} C_{\alpha \sigma}^{\dagger} C_{\alpha^{\prime} \sigma^{\prime}}^{\dagger} C_{\beta^{\prime} \sigma^{\prime}} C_{\beta \sigma},
$$

where $C_{\alpha \sigma}^{\dagger}\left(C_{\alpha \sigma}\right)$ creates (destroys) an electron in molecular orbital $\psi_{\alpha}$ in subspace $\mathrm{C}$ with spin $\sigma, N_{\alpha}=\sum_{\sigma} C_{\alpha \sigma}^{\dagger} C_{\alpha \sigma}$, and $\tilde{U}_{\alpha \alpha^{\prime} \beta \beta^{\prime}}$ is the Coulomb matrix. Note that the Hubbard interaction, which is local in the site basis, becomes more complicated in the basis of the molecular orbitals, $\tilde{U}_{\alpha \alpha^{\prime} \beta \beta^{\prime}}=$ $U \sum_{i} \psi_{\alpha}^{*}(i) \psi_{\alpha^{\prime}}^{*}(i) \psi_{\beta}(i) \psi_{\beta^{\prime}}(i)$. On the other hand, the coupling to the substrate, described by the hybridization function, is still the same for all ZMs and constant, i.e., $\Delta_{\alpha}(\omega)=-i \Gamma$, since we have assumed it to be site-independent. $\varepsilon_{\mathrm{C}}$ in (3) is the effective single-particle energy of the $\mathrm{ZMs}$, related to the on-site energy $\epsilon_{0}$ of the NG, cf. Eq. (2), but modified by the mean-field interaction of $\mathrm{C}$ with the rest of the NG. $\varepsilon_{\mathrm{C}}$ is chosen such that $\mathrm{C}$ is close to half-filling, i.e., close to the particle-hole $(\mathrm{ph})$ symmetric point corresponding to the neutral isolated $\mathrm{NG}$, given by $\varepsilon_{\mathrm{C}}^{*}=-\tilde{U} / 2-\left(\tilde{U}^{\prime}-\tilde{J}_{\mathrm{H}} / 2\right)\left(N_{\mathrm{C}}-\right.$ 1) [41], where $\tilde{U}=\tilde{U}_{\alpha \alpha \alpha \alpha}$ is the intraorbital Coulomb, $\tilde{U}^{\prime}=$ $\tilde{U}_{\alpha \beta \alpha \beta}$ is the interorbital Coulomb, and $\tilde{J}_{\mathrm{H}}=\tilde{U}_{\alpha \beta \beta \alpha}$ is the Hund interaction between the ZMs $\alpha \neq \beta$ in C. Charge transfer from the substrate leads to detuning of $\epsilon_{\mathrm{C}}$ from the ph symmetric point. In our model, the detuning from ph symmetry, $\delta \varepsilon_{\mathrm{C}}=\varepsilon_{\mathrm{C}}-\varepsilon_{\mathrm{C}}^{*}$, is taken as an adjustable parameter.

We solve the multiorbital AIM within the one-crossing approximation (OCA) [50,55]. In short, OCA consists in a diagrammatic expansion of the propagators $G_{m}(\omega)$ associated with the many-body eigenstates [or pseudoparticles (PPs)] $|m\rangle$ with eigenenergies $E_{m}$ of the isolated impurity Hamiltonian (3) in terms of the hybridization function $\Delta_{\alpha}(\omega)$ to infinite order, but summing only a subset of diagrams (only those where conduction electron lines cross at most once). The PP self-energy $\Sigma_{m}(\omega)$ describes the interaction of the PP $m$ with other PPs via the conduction electron bath. Its real part yields the renormalization of the many-body eigenenergies $E_{m}$ and correspondingly of the electronic excitations due to coupling to the bath. The electronic spectral functions $A_{\alpha}(\omega)$ for the NG ZMs are then obtained from convolutions of the PP propagators $G_{m}(\omega)$; see Appendix for more details.

In the ideal STM limit (vanishing coupling to the STM tip), the NG flake is in equilibrium with the substrate. Making use of the Meir-Wingreen formula [56] a direct relation between the differential conductance from the tip to the sample and the equilibrium spectral function matrix $\mathbf{A}(\omega)$ of the NG can be derived $[51,57]$. In the zero-temperature limit, this yields

$$
\frac{d I}{d V} \sim \operatorname{Tr}\left[\Gamma^{\mathrm{tip}} \mathbf{A}(e V)\right]=\sum_{\alpha} \gamma_{\alpha}^{\mathrm{tip}} A_{\alpha}(e V)
$$

where $\Gamma^{\text {tip }}$ is the coupling matrix between the NG and the STM tip, and $\mathbf{A}(\omega)$ is the spectral function matrix of the NG. In the last step, we have further taken into account that for our model the spectral function matrix is diagonal in the molecular orbitals $\psi_{\alpha}$, i.e., $\mathbf{A}(\omega)=\sum_{\alpha}\left|\psi_{\alpha}\right\rangle A_{\alpha}(\omega)\left\langle\psi_{\alpha}\right|$, and $\gamma_{\alpha}^{\text {tip }} \equiv\left\langle\psi_{\alpha}\left|\boldsymbol{\Gamma}^{\text {tip }}\right| \psi_{\alpha}\right\rangle$ is the coupling of orbital $\psi_{\alpha}$ to the STM tip.

If the STM tip is directly above a carbon site $i$, the coupling will be mainly to this site, i.e., $\Gamma^{\mathrm{tip}} \approx \gamma_{i}^{\mathrm{tip}}|i\rangle\langle i|$, so that the $d I / d V$ corresponds to the local spectral function $\rho_{i} \equiv\langle i|\mathbf{A}| i\rangle$ of that carbon site $i$, i.e., $d I / d V \sim \gamma_{i}^{\text {tip }} \rho_{i}(e V)$, where the local spectral function can be calculated as

$$
\rho_{i}(\omega)=\sum_{\alpha \in \mathrm{NG}}\left|\psi_{\alpha}(i)\right|^{2} A_{\alpha}(\omega)
$$

Note that the orbital index $\alpha$ runs over all orbitals $\psi_{\alpha}$ of the NG and not just over the ZMs.

In an STM experiment, measuring the map $\left.\mathcal{G}(\mathbf{R}) \equiv \frac{d I}{d V}\right|_{V_{0} ; \mathbf{R}}$ of a molecule for different positions $\mathbf{R}$ of the STM tip and at fixed voltage $V_{0}=\omega_{0} / e$ corresponding to a feature in the $d I / d V$ spectrum, e.g., a Kondo peak, an inelastic step, or HOMO/LUMO resonance, gives an (approximate) picture of the orbital associated with that feature. This $\mathcal{G}(\mathbf{R})$ map roughly corresponds to the spatially resolved spectral function $\rho(\mathbf{r} ; \omega) \equiv\langle\mathbf{r}|\mathbf{A}| \mathbf{r}\rangle$ evaluated at $\omega=\omega_{0}$ and $\mathbf{r}=\mathbf{R}$, which can be calculated easily within our approach as

$$
\rho(\mathbf{r} ; \omega)=\sum_{i, j} \phi_{i}^{*}(\mathbf{r}) \rho_{i j}(\omega) \phi_{j}(\mathbf{r})
$$

where now also the off-diagonal elements of the spectral function in the site basis, $\rho_{i j}(\omega) \equiv\left\langle\phi_{i}|\mathbf{A}(\omega)| \phi_{j}\right\rangle=$ $\sum_{\alpha} \psi_{\alpha}(i) A_{\alpha}(\omega) \psi_{\alpha}^{*}(j)$, are required. Here we assume a single Slater-type $2 p_{z}$-orbital localized at each carbon site of our tight-binding model using Slater's original parametrization [58], i.e., $\phi_{i}(\mathbf{r}) \sim z e^{-\zeta\left|\mathbf{r}-\mathbf{R}_{i}\right|}$, where $\mathbf{r}=(x, y, z), \zeta=$ $1.625 / a_{0}$ for carbon, where $a_{0}=0.5292 \AA$ is the Bohr radius and $\mathbf{R}_{i}=\left(X_{i}, Y_{i}, 0\right)$ is the position of carbon site $i$ (assuming the NG is located in the $z=0$ plane). The carbon sites of the NG flakes are assumed to be at the positions of a perfect graphene lattice with nearest-neighbor distance $a=1.42 \AA$. 

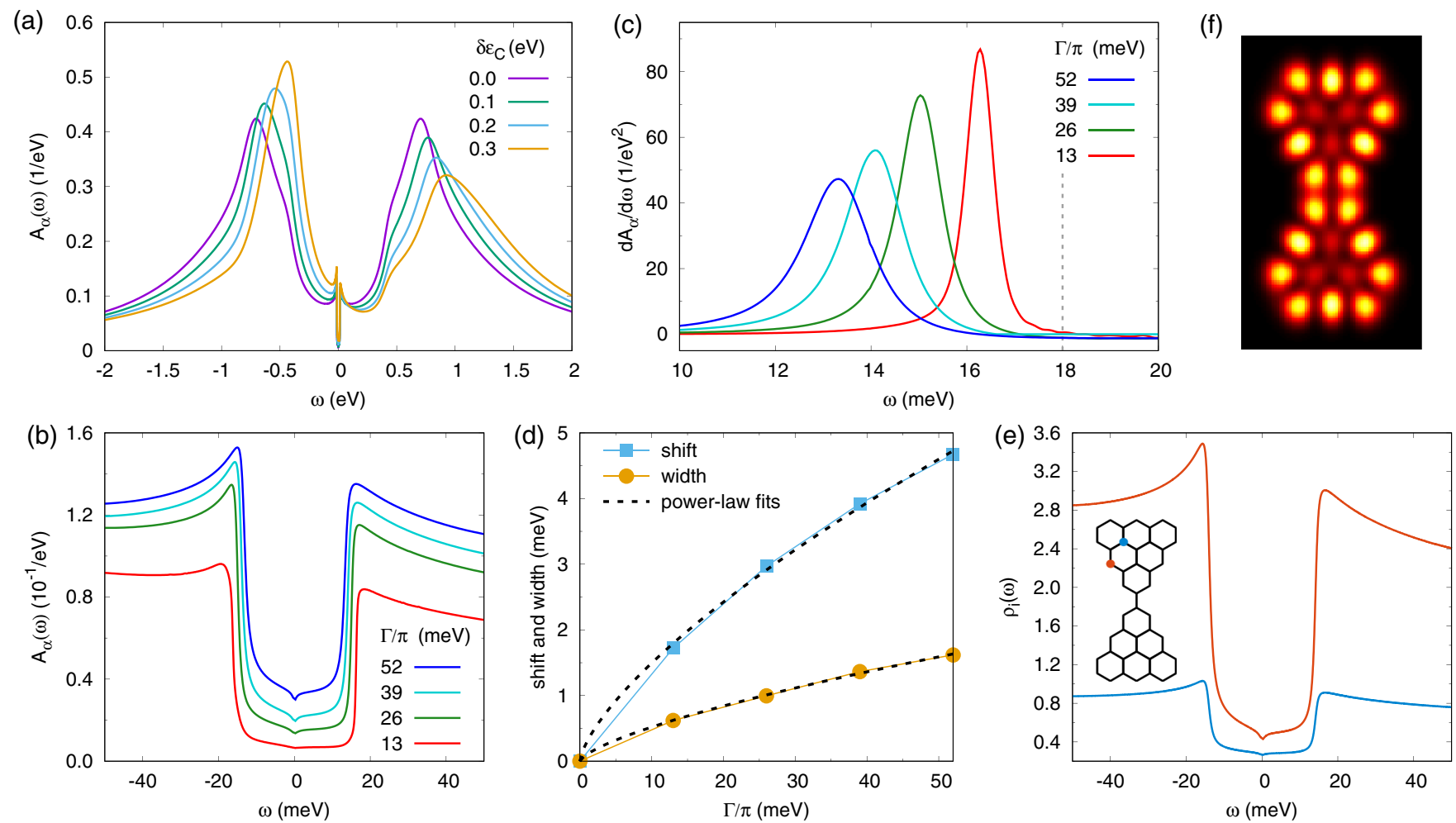

FIG. 2. OCA results for triangulene dimer for $t=-2.7 \mathrm{eV}, U=1.9|t|$, and $\varepsilon_{\mathrm{C}}^{*}=-0.485 \mathrm{eV}$. (a) Spectral function of zero modes for different values of the on-site energy shift $\delta \varepsilon_{\mathrm{C}}$ and for broadening $\Gamma / \pi=52 \mathrm{meV}$. (b) Spectral function at low energies for different values of the broadening $\Gamma$ and for $\delta \varepsilon_{\mathrm{C}}=0.2 \mathrm{eV}$. (c) Derivatives of spectral functions shown in (b) close to the inelastic spin excitation step. The dashed gray line marks the position of the bare excitation energy. (d) Shift and width of the ISTS step feature as a function of the broadening $\Gamma$ for $\delta \varepsilon_{\mathrm{C}}=0.2 \mathrm{eV}$. The dashed lines show power-law fits to the data, which yield exponents of $\alpha \sim 0.7$ for both shift and width as a function of $\Gamma$. (e) Local spectral functions $\rho_{i}(\omega)$ for the two carbon sites of the NG shown in the inset for $\Gamma / \pi=39 \mathrm{meV}$ and $\delta \varepsilon_{\mathrm{C}}=0.2 \mathrm{eV}$. (f) Density map of spatially resolved spectral function $\rho\left(\mathbf{r} ; \omega_{-}\right)$evaluated at $z=5 \AA$ above the molecular plane for $\omega_{-}=-15.5$ meV. Other parameters as in (e).

\section{RESULTS}

\section{A. Triangulene dimer}

First, we consider a NG consisting of two coupled triangulenes as shown schematically in Fig. 1(a). Such triangulene dimers deposited on $\mathrm{Au}$ substrates were recently studied experimentally by STM spectroscopy, and theoretically by configuration interaction of an isolated molecule described by the Hubbard model (2) [17]. Here we investigate the effect of the substrate on the $d I / d V$ and $d^{2} I / d V^{2}$ spectra, and in particular the renormalization of the spin excitation energies due to the coupling to the substrate.

The previous study showed that each of the triangulene units of the dimer carries a spin-1 located in its two ZMs due to strong intratriangulene Hund's rule coupling $J_{\mathrm{H}}[8]$. On the other hand, a Coulomb-driven kinetic exchange mechanism involving the virtual occupation (deoccupation) of the empty (filled) molecular orbitals closest in energy to the ZMs gives rise to an effective antiferromagnetic coupling $J_{\text {eff }} \ll J_{\mathrm{H}}$ between the spins $S=1$ of the individual triangulenes. $J_{\text {eff }}$ quenches the total spin of the dimer, leading to an $S=0$ singlet GS, separated in energy by $J_{\text {eff }}$ from an excited $S=1$ triplet state, and by $3 J_{\text {eff }}$ from an $S=2$ quintuplet state [17].

Here we only take into account the four ZMs of the two triangulene units and model the effective antiferromag- netic exchange interaction between the two triangulenes by a Heisenberg exchange interaction $\hat{H}_{S}=J_{\text {eff }} \mathbf{S}_{1} \cdot \mathbf{S}_{2}$. We take the value for $J_{\text {eff }}$ from the energy gap between the $S=0$ GS and the excited $S=1$ state of a configuration interaction calculation including the two molecular orbitals of the dimer closest in energy to the zero modes. Assuming a Hubbard interaction of $U=1.9|t|=5.13 \mathrm{eV}$ results in $J_{\mathrm{eff}}=18 \mathrm{meV}$.

Figure 2 shows results for the dimer model calculated within OCA for different coupling strengths and on-site energies $\varepsilon_{\mathrm{C}}$ at low temperature $(k T=0.1 \mathrm{meV} \sim 1.2 \mathrm{~K})$. Panel (a) shows the effect of detuning, $\delta \varepsilon_{\mathrm{C}}=\varepsilon_{\mathrm{C}}-\varepsilon_{\mathrm{C}}^{*}$, of the on-site energy $\varepsilon_{\mathrm{C}}$ away from the particle-hole (ph) symmetric point, $\varepsilon_{\mathrm{C}}^{*}=-485 \mathrm{meV}$, on the spectral function on a large energy scale, including the Coulomb peaks. As the detuning $\delta \varepsilon_{\mathrm{C}}$ increases, the spectra become more asymmetric: the lower Coulomb peak (corresponding to the electron removal energy) moves closer to the Fermi level, while the upper peak (electron addition energy) moves farther away from it. As expected, the value of the Coulomb gap of $\sim 1.4 \mathrm{eV}$ in our model slightly underestimates the experimentally measured value of about $\sim 1.65 \mathrm{eV}$ due to the neglect of the long-range part of the Coulomb interaction. A phenomenological way to fix the Coulomb gap was given in Ref. [26].

We now focus on the low-energy part of the spectra, specifically on the lowest spin excitation. We choose a detuning of 
$\delta \varepsilon_{\mathrm{C}}=0.2 \mathrm{eV}$, which brings the lower Coulomb peak approximately into the same position as the experimentally observed one at about $-0.4 \mathrm{eV}$. Figure 2(b) shows the spectral function for this choice of $\varepsilon_{\mathrm{C}}$ and for different values of the broadening by the substrate $\Gamma$. The spectral functions show two steps (one at negative and one at positive energy) similar to those found in the experiment [17]. These steps correspond to inelastic spin excitations from the $S=0$ singlet GS to the excited $S=1$ triplet state. As the single-particle broadening $\Gamma$ increases, the inelastic steps move inward to lower energies, due to the renormalization induced by the coupling to the substrate, departing from the bare excitation energy of $\sim 18 \mathrm{meV}$, and their triangular shape associated with Kondo exchange coupling becomes more pronounced.

The renormalization of the excitation energies due to the coupling to the bath can be better appreciated in the derivative of the spectral function [Fig. 2(c)], which shows a peak at the excitation energy. Clearly, the peak moves to lower energies and broadens, as the coupling to the bath $\Gamma$ increases. Figure 2(d) shows the shift of the excitation energy and the broadening of the step extracted from the derivative of the spectral function [Fig. 2(c)] as a function of $\Gamma$. Interestingly, we find a power-law behavior $\sim \Gamma^{0.7}$ for both the shift and width of the step. At first glance this seems to contradict second-order perturbation theory results for the similar case of renormalization of the single-ion magnetic anisotropy of single magnetic adatoms on conducting substrates, which predict a quadratic behavior $[38,39]$. However, the perturbation theory results are expected to be valid only in the weakcoupling (small $\Gamma$ ) regime to which we do not have access due to numerical issues [59]. It is therefore conceivable that the power-law behavior turns into quadratic behavior for very small $\Gamma$.

Importantly, our results show that the bare spin excitation energies are always renormalized due to the coupling of the NG to the conduction electron bath in the substrate. The magnitude of the renormalization is, according to our calculations, larger than the broadening of the spectral function peak. This is similar to the renormalization of the single-ion magnetic anisotropy measured in STM spectroscopy of single magnetic atoms and molecules on conducting substrates [38]. In this sense, the excitation energy measured in an STM experiment is never really the intrinsic one of the NG since coupling to the conduction electrons in the substrate cannot be avoided in an STM setup. Introducing insulating layers between the NG and the conducting substrate in order to decouple the NG from the conduction electrons can significantly reduce this effect, and thus allow us to measure the bare excitation energies to a better approximation.

We now investigate the spatial dependence of the spectra. Since the triangulene ZMs are mainly localized at the edge atoms of the NGs (cf. Fig. 1), we expect that the spectral signature of the inelastic spin excitations is localized there. This is indeed the case, as can be seen in Fig. 2(e), which shows the local spectral density $\rho_{i}(\omega)$ for two carbon sites, one at the edge and one in the interior of the NG. Both show inelastic spin excitation steps, but the amplitude for the border site is significantly larger than for the one in the interior by almost one order of magnitude. Figure 2(f) shows a density map of the spatially resolved spectral function $\rho(\mathbf{r} ; \omega)$ evaluated at the energy of the maximum of the left step, $\omega_{-}=15 \mathrm{meV}$. Clearly, the density map resembles the spatial distribution of the ZMs (cf. Fig. 1).

\section{B. Triangulene monomer}

Next we consider a single triangulene molecule, shown in Fig. 1(c), i.e., the basic building block of the triangulene dimer considered in the previous section. Since now the antiferromagnetic exchange between the triangulene units is absent, the spin $S=1$ carried by the two ZMs of the triangulene remains unquenched. Our results show a Kondo peak. We note that Kondo peak measurements have been reported in at least two spin-1 NGs on metallic substrates [19,21], but not on triangulenes. However, so far STM spectra of single triangulene molecules have only been measured for nonmetallic $\mathrm{Xe}(111)$ as substrate, and no Kondo effect was found in this case. This is to be expected as the coupling to the sample electrode via the "insulating" Xe layers or to the tip electrode via the vacuum is very weak. Due to exponential decay of the Kondo temperature with the ratio $\delta U / \Gamma$ (where $\delta U$ is the charging energy), $T_{K} \sim e^{-\delta U / \Gamma}$, it becomes vanishingly small for $\Gamma \ll \delta U$. Moreover, according to Nevidomskyy and Coleman, for systems with $S>1 / 2$ the Kondo temperature is exponentially reduced compared to a spin- $1 / 2$ Kondo system [60].

Nevertheless, if the coupling to the conduction electrons becomes sufficiently strong, for example for a triangulene on a metallic substrate, the Kondo effect may occur at experimentally accessible temperatures. Also, on a metallic substrate the Coulomb interaction $U$ will be reduced due to screening by the conduction electrons, thus further enhancing the Kondo temperature. In Fig. 3(b) we show the effect of changing the coupling to the substrate $\Gamma$ while keeping the Coulomb interaction fixed to $U=|t|$. Panel (a) shows the spectral function of the ZMs for different values of $\Gamma$ at low temperature $T \sim 5 \mathrm{~K}$ : The spectra show lower and upper Coulomb peaks at $\omega \sim \pm 300 \mathrm{meV}$ and a Kondo peak at $\omega=0$. As $\Gamma$ is increased, the Coulomb peaks broaden and the Kondo peak becomes more pronounced. The latter can be better appreciated in panel (b), which shows the evolution of the Kondo peak as $\Gamma$ changes. For the largest coupling shown in Fig. 3 $(\Gamma / \pi=26 \mathrm{meV})$, we obtain a Kondo temperature estimated from the half-width of the Kondo peak of about $T_{K} \sim 15 \mathrm{~K}$, well above typical temperatures that can be achieved in STM experiments. Also note that the critical magnetic field for observing the Zeeman splitting of the Kondo peak in the $S=1$ Kondo case is given by $B_{c} \sim k T_{K} / 2 \mu_{B}$. Thus for Kondo temperatures of up to $15 \mathrm{~K}$ obtained here, fields of up to $11 \mathrm{~T}$ are required to observe the splitting experimentally. For the lowest $\Gamma$ considered here, the Kondo peak vanishes at the chosen temperature $T \sim 5 \mathrm{~K}$. We note that inelastic steps, corresponding to transitions from $S=1$ to 0 , with energies in the range of the intramolecular exchange of about $260 \mathrm{meV}$, cf. Figs. 6c,d in Ref. [26], are not visible in the spectra. They are obscured by the much more pronounced Coulomb peaks, which happen to reside just at the same energy. 


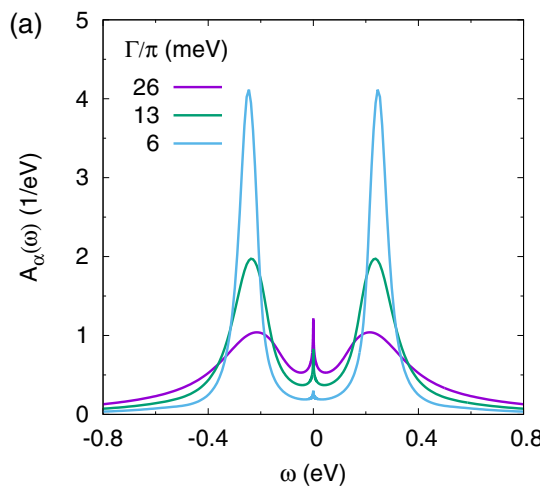

(e)

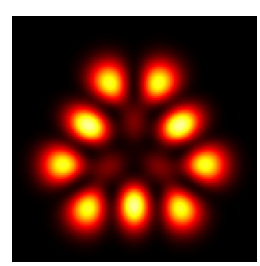

(d)

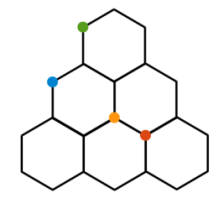

(b)

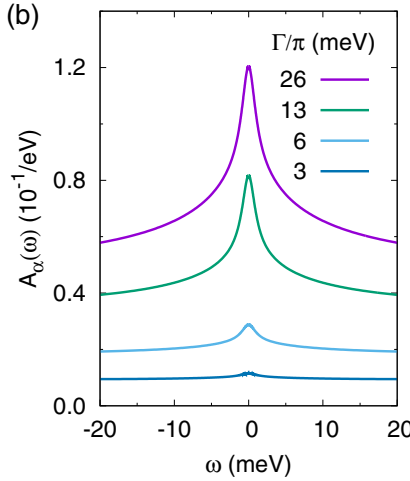

(c) 0.3

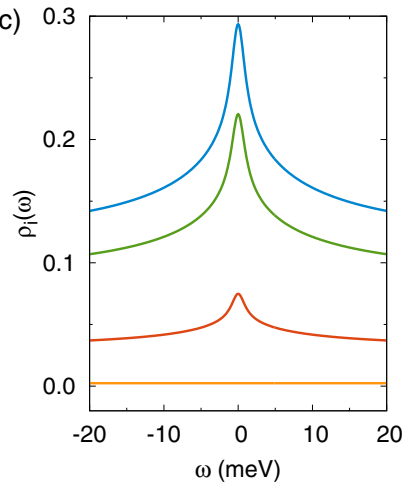

FIG. 3. Spectral functions calculated within OCA for the triangulene monomer for $U=|t|$ and $t=-2.7 \mathrm{eV}$ at ph symmetry $\left(\varepsilon_{\mathrm{C}}=\right.$ $-0.26 \mathrm{eV}$ ). (a) Spectral function of ZMs for different values of the coupling to the substrate $\Gamma$ at low temperature $k T=0.4 \mathrm{meV} \sim 5 \mathrm{~K}$. (b) Same as (a) but for a smaller energy scale showing the evolution of the Kondo peak as $\Gamma$ decreases. (c) Local spectral function for the carbon sites marked in the atomic structure (d) in the corresponding color. (e) Density map of spatially resolved spectral function $\rho\left(\mathbf{r} ; \omega_{0}\right)$ evaluated at $z=5 \AA$ above the molecular plane for $\omega_{0}=0$.

Figure 3(c) shows the local spectral functions $\rho_{i}(\omega)$ projected on different carbon sites $i$ marked by circles in corresponding colors in the atomic structure in panel (d). In correspondence to the weight of the site in the $\mathrm{ZM}$ wave function $\psi_{\alpha}(i)$, cf. Fig. 1(c), the Kondo peak becomes less and less pronounced as we move from the blue to the green and to the red site. On the other hand, the Kondo peak is completely absent on the orange site, which belongs to a different sublattice than the ZMs. The density map in Fig. 3(e) shows the spatially resolved spectral function evaluated at $\omega=0$. As the Kondo peak only shows up at the sites contributing to the $\mathrm{ZMs}$, the map reflects the density associated with the wave functions of the ZMs; cf. Fig. 1(c).

\section{C. "Rocket" structure}

Finally, we consider the "rocket" structure shown in Fig. 1(e), which has recently been studied experimentally by STM spectroscopy [21]. As reported there, we also find a spin1 Kondo effect signaled by a sharp Abrikosov-Suhl resonance at the Fermi level at low temperatures; see Fig. 4(a). Similar to triangulene discussed in the previous section, the "rocket" structure hosts a spin-1 in its two ZMs $\psi_{1}$ and $\psi_{2}$. However, different from triangulene, $\psi_{1}$ and $\psi_{2}$ have very different
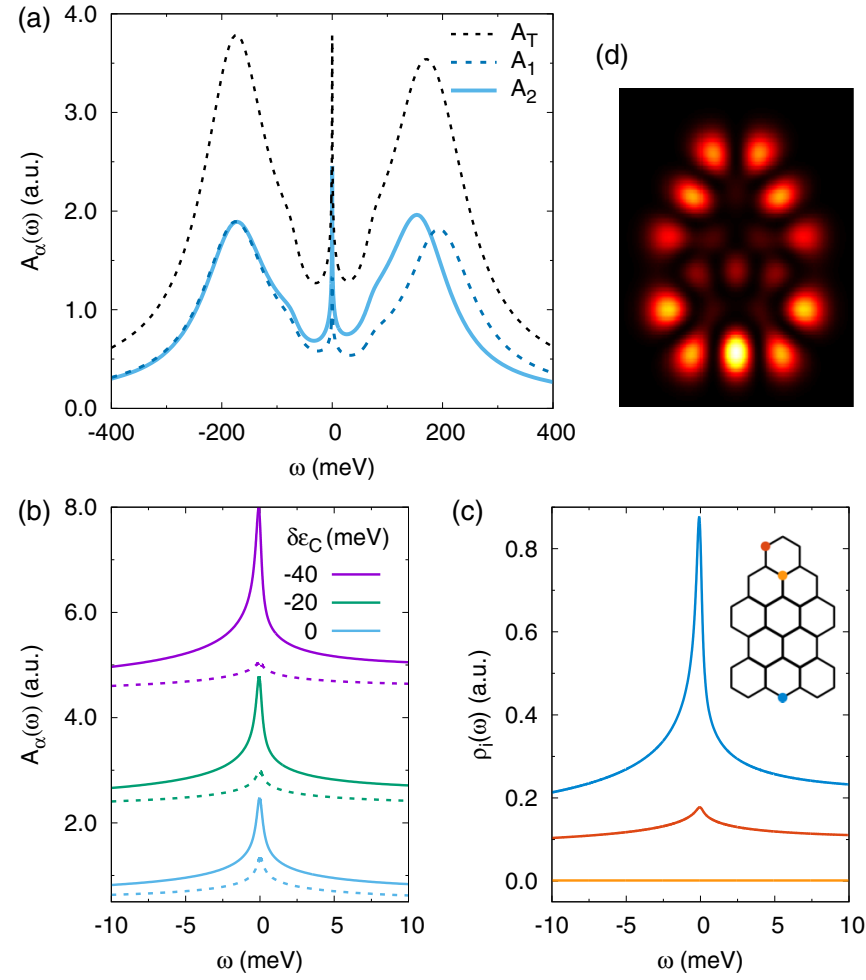

FIG. 4. Spectral functions calculated within the OCA for rocket structure for $U=|t|$ and $\Gamma / \pi=13 \mathrm{meV}$ at low temperature, $k T=$ $0.1 \mathrm{meV} \sim 1.2 \mathrm{~K}$. (a) Orbital-resolved $\left(A_{1}, A_{2}\right)$ and total $\left(A_{\mathrm{T}}=\right.$ $\left.A_{1}+A_{2}\right)$ spectral functions at half-filling $\left(N=N_{1}+N_{2}=2\right)$ at $\varepsilon_{\mathrm{C}}=-0.186 \mathrm{eV}$. (b) Orbital-resolved spectral functions around the Kondo peak for different values of detuning $\delta \varepsilon_{\mathrm{C}}$ from half-filling. The dashed (solid) lines show the spectral functions of orbital 1 and 2 , respectively. (c) Local spectral function $\rho_{i}(\omega)$ for carbon sites $i$ marked in corresponding color in the atomic structure shown in the inset for $\delta \varepsilon_{\mathrm{C}}=-40 \mathrm{meV}$. (d) Density map of spatially resolved spectral function $\rho\left(\mathbf{r} ; \omega_{0}\right)$ evaluated at $z=5 \AA$ above the molecular plane for $\omega_{0}=0$.

densities, with $\psi_{1}$ mostly localized in the upper part and $\psi_{2}$ mostly localized in the lower part of the NG, as can be seen in Fig. 1(e). Interestingly, $\psi_{2}$ is also somewhat more delocalized than $\psi_{1}$. This leads to a slightly smaller intraorbital Coulomb repulsion for the former $\left(U_{2} \sim 300 \mathrm{meV}\right)$ than for the latter $\left(U_{1} \sim 350 \mathrm{meV}\right)$, as these matrix elements are inversely proportional to extension of the orbitals [8]. Accordingly, the upper Coulomb peaks of both orbitals corresponding to the addition of one electron over the half-filled $(N=2)$ GS are split in energy by $\sim U_{1}-U_{2} \sim 50 \mathrm{meV}$, as can be seen in Fig. 4(a). On the other hand, the lower Coulomb peak is at the same position for both orbitals due to the degeneracy of their energy levels.

As the addition energies $\delta U_{\alpha}$ differ for both orbitals, so do their respective Kondo temperatures, according to $T_{K, \alpha} \sim$ $e^{-\delta U_{\alpha} / \Gamma}$. Taking into account that the broadening $\Gamma$ is equal for both orbitals, we thus have $T_{K, 1}<T_{K, 2}$. Indeed the Kondo peak for $\psi_{1}$ is considerably less pronounced than the one for $\psi_{2}$, as can be seen in Fig. 4(b). Moreover, moderately lowering the energy levels of both orbitals, the disparity of both Kondo peaks is further enhanced for the following 
reason: At half-filling $N \sim 2$, the individual occupations of both orbitals are slightly different due to their different Coulomb interactions, i.e., $\psi_{1}$ is slightly less than half-filled, $N_{1} \sim 0.99$, while $\psi_{2}$ is slightly more than half-filled, $N_{2} \sim$ 1.01. Upon lowering the energy of both orbitals, $N_{1}$ is at first driven closer to half-filling, while $N_{2}$ is driven farther away from half-filling. Additionally, the higher charging energy of $\psi_{1}$ leads to $N_{1}$ increasing slower than $N_{2}$ upon lowering of the energy levels. Thus charge fluctuations and correspondingly the Kondo temperature are reduced for orbital $\psi_{1}$, while they are enhanced for orbital $\psi_{2}$. For temperatures $T_{K, 1}<T<T_{K, 2}$ this leads to partial Kondo screening where only the spin- $1 / 2$ in $\psi_{2}$ is screened. For the largest detuning considered here $\left(\delta_{\mathrm{C}}=-40 \mathrm{meV}\right)$ we estimate $T_{K, 2} \sim 7.5 \mathrm{~K}$ from the halfwidth of the Kondo peak of orbital $\psi_{2}$, in very good agreement with the experimental value obtained by $\mathrm{Li}$ and co-workers [21].

As the two ZMs are localized in different parts of the NG, the different manifestation of the Kondo peaks in the two orbitals leads to strongly site-dependent spectral functions, as can be seen in Fig. 4(c): While sites in the lower part of the NG corresponding to $\psi_{2}$ (e.g., the blue site) show a very pronounced Kondo peak, the Kondo peak is strongly suppressed for sites in the upper part (e.g., the red site) of the NG corresponding to $\psi_{1}$. On the other hand, sites that live on a different sublattice than the ZMs (e.g., the orange site) do not show a Kondo peak at all. The density map of the spatially resolved spectral function $\rho\left(\mathbf{r} ; \omega_{0}=0\right)$ summarizes these findings: due to the predominance of the Kondo peak in the lower part of the NG, the density map is considerably brighter in the lower part than in the upper part. On the other hand, the inner part of the NG remains mostly dark as the ZMs predominantly live at the border of the NG.

\section{CONCLUSIONS}

We have studied the effect of coupling to a metallic substrate on the spectra of open-shell NGs. For NGs with a nondegenerate $S=0 \mathrm{GS}$ such as the triangulene dimer, the exchange coupling to the conduction electrons in the substrate leads to a reduction of the energies of the $S=0 \rightarrow S=1$ excitations, compared with the bare energies computed ignoring the coupling to the substrate. On the other hand, for NGs with a degenerate GS, coupling to the conduction electrons leads to the Kondo effect, fully or partially screening the spin of the $\mathrm{NG}$, if the coupling is strong enough and/or the temperature is low enough. In very symmetric situations such as in the triangulene molecule, the Kondo screening is equally strong for all ZMs, generally leading to a situation in which the entire spin $S=1$ of the NG is screened by the Kondo effect. In this case, the Kondo temperature is exponentially reduced compared to the spin-1/2 Kondo effect [60], thus requiring very low temperatures and/or very strong coupling to the substrate to become measurable. For less symmetric situations such as the "rocket" structure, the Kondo screening strength may vary considerably among the ZMs due to their different intraorbital Coulomb interactions, leading to partial Kondo screening of the NG spin as reported in recent experimental work [21]. Our results further indicate that in this case the partial Kondo screening situation leads to a manifestation of the Kondo peak predominantly at carbon sites in the lower part of the NG associated with the Kondo screened ZM.

Our results stress the need to consider the coupling to the substrate in order to account for IETS experimental results, and to anticipate discrepancies with high-level quantum chemistry calculations for gas-phase molecules. Our formalism also predicts the broadening of the spectral features that ultimately reflects the finite lifetime of the spin excitations due to Kondo exchange with the substrate, and it will have to be considered in proposals to use nanographenes for quantum information purposes [61,62], whenever nanographenes are to be contacted to electrodes.

\section{ACKNOWLEDGMENTS}

D.J. acknowledges funding by the Basque Government through grant "Grupos Consolidados UPV/EHU del Gobierno Vasco" (Grant No. IT1249-19). R.O. acknowledges financial support from Generalitat Valenciana and Fondo Social Europeo (Grant No. ACIF/2018/175). J.F.R. acknowledges financial support from Generalitat Valenciana (Prometeo2017/139), and MINECO-Spain (Grant No. PID2019-109539GB-C41). J.F.R. and D.J. acknowledge funding from FEDER/Junta de Andalucía-Consejería de Transformación Económica, Industria, Conocimiento y Universidades, Grant No. P18-FR-4834. We acknowledge fruitful discussions with G. Catarina and J. C. Sancho-García.

\section{APPENDIX: ONE-CROSSING APPROXIMATION}

We solve the multiorbital AIM within the one-crossing approximation (OCA) [50,55]. The first step is a numerical diagonalization of the isolated impurity Hamiltonian (3), $\mathcal{H}_{\mathrm{C}}|m\rangle=E_{m}|m\rangle$, for different fillings $N=\sum_{\alpha}\left\langle N_{\alpha}\right\rangle$ of the impurity shell. Here we consider the NG close to half-filling. The coupling to the substrate $\mathcal{V}_{\text {hyb }}$ only connects eigenstates with occupations differing by one electron, leading to charge and spin fluctuations in the impurity shell. Thus for a NG with $M$ ZMs we consider the occupations $N=M$ and $N=M \pm 1$. It is the fluctuations between the impurity GS manifold and excited states with one more or one fewer electron that give rise to both the Kondo effect and renormalization of the spin excitation energies.

In the next step, a diagrammatic expansion of the manybody eigenstates $|m\rangle$ of the (isolated) impurity $\mathcal{H}_{\mathrm{C}}$ in terms of the hybridization with the substrate is developed. To this end, one introduces so-called pseudoparticles (PPs) $m$ corresponding to the many-body eigenstates $|m\rangle$. The full propagator of such a PP $m$ can be written as $G_{m}(\omega)=1 /\left[\omega-\lambda-E_{m}-\right.$ $\left.\Sigma_{m}(\omega)\right]$, where $\Sigma_{m}(\omega)$ is the PP self-energy which describes the renormalization (real part) and broadening (imaginary part) of the PP $m$ due to the interaction with other PPs $m^{\prime}$ mediated by the conduction electron bath. $-\lambda$ is the chemical potential for the PPs, which has to be adjusted such that the total PP charge $Q=\sum_{m} a_{m}^{\dagger} a_{m}$ is conserved, imposing the completeness of the many-body Hilbert space.

The OCA consists in a diagrammatic expansion of the PP self-energies $\Sigma_{m}$ in terms of the hybridization function $\Delta_{\alpha}(\omega)$ to infinite order but summing only a subset of diagrams (only those involving conduction electron lines crossing at most 
once). This leads to a set of coupled integral equations for the PP propagators and self-energies that have to be solved self-consistently.

Once the OCA equations are solved, the real electron spectral function $A_{\alpha}(\omega)$ for the NG ZMs is obtained from convolutions of PP propagators $G_{m}(\omega)$. The PP resonances for the GS manifold feature sharp resonances at the renormalized many-body energies $E_{m}^{*}=E_{m}+\operatorname{Re} \Sigma_{m}\left(E_{m}^{*}\right)$. The differences between the renormalized energies of the excited states $E_{m}^{*}$ and the GS $E_{0}^{*}$ yield the actual electronic excitations of the system. Thus we see that the coupling to the conduction electron bath leads to the renormalization of the spin excitations, described by the real part of the PP self-energies within our approach. More details on the application of the OCA method to nanoscale quantum magnets can be found, e.g., in Refs. [40,41].
[1] H. C. Longuet-Higgins, J. Chem. Phys. 18, 265 (1950).

[2] E. Clar and C. Mackay, Tetrahedron 28, 6041 (1972).

[3] W. T. Borden and E. R. Davidson, J. Am. Chem. Soc. 99, 4587 (1977).

[4] J. Fernández-Rossier and J. J. Palacios, Phys. Rev. Lett. 99, 177204 (2007).

[5] W. L. Wang, O. V. Yazyev, S. Meng, and E. Kaxiras, Phys. Rev. Lett. 102, 157201 (2009).

[6] Y. Morita, S. Suzuki, K. Sato, and T. Takui, Nat. Chem. 3, 197 (2011).

[7] M. Melle-Franco, Chem. Commun. 51, 5387 (2015).

[8] R. Ortiz, R. Á. Boto, N. García-Martínez, J. C. Sancho-García, M. Melle-Franco, and J. Fernández-Rossier, Nano Lett. 19, 5991 (2019).

[9] A. Narita, X. Feng, and K. Müllen, Chem. Rec. 15, 295 (2015).

[10] P. Ruffieux, S. Wang, B. Yang, C. Sánchez-Sánchez, J. Liu, T. Dienel, L. Talirz, P. Shinde, C. A. Pignedoli, D. Passerone, T. Dumslaff, X. Feng, K. Müllen, and R. Fasel, Nature (London) 531, 489 (2016).

[11] S. Song, J. Su, M. Telychko, J. Li, G. Li, Y. Li, C. Su, J. Wu, and J. Lu, Chem. Soc. Rev. 50, 3238 (2021).

[12] S. Wang, L. Talirz, C. A. Pignedoli, X. Feng, K. Müllen, R. Fasel, and P. Ruffieux, Nat. Commun. 7, 11507 (2016).

[13] S. Mishra, D. Beyer, K. Eimre, S. Kezilebieke, R. Berger, O. Gröning, C. A. Pignedoli, K. Müllen, P. Liljeroth, P. Ruffieux et al., Nat. Nanotechnol. 15, 22 (2019).

[14] S. Mishra, X. Yao, Q. Chen, K. Eimre, O. Groening, R. Ortiz, M. Di Giovannantonio, J. C. Sancho-Garcia, J. FernandezRossier, C. A. Pignedoli, K. Müllen, P. Ruffieux, A. Narita, and R. Fasel, Nat. Chem. 13, 581 (2021).

[15] N. Pavliček, A. Mistry, Z. Majzik, N. Moll, G. Meyer, D. J. Fox, and L. Gross, Nat. Nano. 12, 308 (2017).

[16] J. Su, M. Telychko, P. Hu, G. Macam, P. Mutombo, H. Zhang, Y. Bao, F. Cheng, Z.-Q. Huang, Z. Qiu, S. J. R. Tan, H. Lin, P. Jelínek, F.-C. Chuang, J. Wu, and J. Lu, Sci. Adv. 5, eaav7717 (2019).

[17] S. Mishra, D. Beyer, K. Eimre, R. Ortiz, J. Fernández-Rossier, R. Berger, O. Gröning, C. A. Pignedoli, R. Fasel, X. Feng et al., Angew. Chem., Int. Ed. 59, 12041 (2020).

[18] S. Mishra, G. Catarina, F. Wu, R. Ortiz, D. Jacob, K. Eimre, J. Ma, C. A. Pignedoli, X. Feng, P. Ruffieux, J. FernandezRossier, and R. Fasel, arXiv:2105.09102.

[19] X. Su, C. Li, Q. Du, K. Tao, S. Wang, and P. Yu, Nano Lett. 20, 6859 (2020).

[20] J. Li, S. Sanz, M. Corso, D. J. Choi, D. Peña, T. Frederiksen, and J. I. Pascual, Nat. Commun. 10, (2019).
[21] J. Li, S. Sanz, J. Castro-Esteban, M. Vilas-Varela, N. Friedrich, T. Frederiksen, D. Peña, and J. I. Pascual, Phys. Rev. Lett. 124, 177201 (2020).

[22] Y. Zheng, C. Li, C. Xu, D. Beyer, X. Yue, Y. Zhao, G. Wang, D. Guan, Y. Li, H. Zheng, C. Liu, J. Liu, X. Wang, W. Luo, X. Feng, S. Wang, and J. Jia, Nat. Commun. 11, 1 (2020).

[23] S. Mishra, D. Beyer, K. Eimre, J. Liu, R. Berger, O. Gröning, C. A. Pignedoli, K. Müllen, R. Fasel, X. Feng, and P. Ruffieux, J. Am. Chem. Soc. 141, 10621 (2019).

[24] A. Sánchez-Grande, J. I. Urgel, L. Veis, S. Edalatmanesh, J. Santos, K. Lauwaet, P. Mutombo, J. M. Gallego, J. Brabec, P. Beran, D. Nachtigallová, R. Miranda, N. Martín, P. Jelínek, and D. Écija, J. Phys. Chem. Lett. 12, 330 (2021).

[25] J. Hieulle, S. Castro, N. Friedrich, A. Vegliante, F. R. Lara, S. Sanz, D. Rey, M. Corso, T. Frederiksen, J. I. Pascual, and D. Peña, arXiv:2107.02198.

[26] R. Ortiz and J. Fernández-Rossier, Prog. Surf. Sci. 95, 100595 (2020).

[27] M. A. Reed, Mater. Today 11, 46 (2008).

[28] A. J. Heinrich, J. A. Gupta, C. P. Lutz, and D. M. Eigler, Science 306, 466 (2004).

[29] C. F. Hirjibehedin, C.-Y. Lin, A. F. Otte, M. Ternes, C. P. Lutz, B. A. Jones, and A. J. Heinrich, Science 317, 1199 (2007).

[30] J. Fernández-Rossier, Phys. Rev. Lett. 102, 256802 (2009).

[31] R. Žitko and T. Pruschke, New. J. Phys. 12, 063040 (2010).

[32] M. Ternes, New J. Phys. 17, 063016 (2015).

[33] N. Tsukahara, K.-i. Noto, M. Ohara, S. Shiraki, N. Takagi, Y. Takata, J. Miyawaki, M. Taguchi, A. Chainani, S. Shin, and M. Kawai, Phys. Rev. Lett. 102, 167203 (2009).

[34] C. F. Hirjibehedin, C. P. Lutz, and A. J. Heinrich, Science 312, 1021 (2006).

[35] A. Spinelli, B. Bryant, F. Delgado, J. Fernández-Rossier, and A. F. Otte, Nat. Mater. 13, 782 (2014).

[36] D.-J. Choi, N. Lorente, J. Wiebe, K. von Bergmann, A. F. Otte, and A. J. Heinrich, Rev. Mod. Phys. 91, 041001 (2019).

[37] R. Korytár, N. Lorente, and J.-P. Gauyacq, Phys. Rev. B 85, 125434 (2012).

[38] J. C. Oberg, M. R. Calvo, F. Delgado, M. Moro-Lagares, D. Serrate, D. Jacob, J. Fernandez-Rossier, and C. F. Hirjibehedin, Nat. Nanotechnol. 9, 64 (2014).

[39] F. Delgado, C. Hirjibehedin, and J. Fernández-Rossier, Surf. Sci. 630, 337 (2014).

[40] D. Jacob and J. Fernández-Rossier, Eur. Phys. J. B 89, 210 (2016).

[41] D. Jacob, Phys. Rev. B 97, 075428 (2018).

[42] A. C. Hewson, The Kondo Problem to Heavy Fermions (Cambridge University Press, Cambridge, 1997).

[43] K. Sengupta and G. Baskaran, Phys. Rev. B 77, 045417 (2008). 
[44] P. S. Cornaglia, G. Usaj, and C. A. Balseiro, Phys. Rev. Lett. 102, 046801 (2009).

[45] T. O. Wehling, A. V. Balatsky, M. I. Katsnelson, A. I. Lichtenstein, and A. Rosch, Phys. Rev. B 81, 115427 (2010).

[46] D. Jacob and G. Kotliar, Phys. Rev. B 82, 085423 (2010).

[47] L. Fritz and V. Vojta, Rep. Prog. Phys. 76, 032501 (2013).

[48] A. Principi, G. Vignale, and E. Rossi, Phys. Rev. B 92, 041107(R) (2015).

[49] Z. Shi, E. M. Nica, and I. Affleck, Phys. Rev. B 100, 125158 (2019).

[50] K. Haule, S. Kirchner, J. Kroha, and P. Wölfle, Phys. Rev. B 64, 155111 (2001).

[51] D. Jacob and S. Kurth, Nano Lett. 18, 2086 (2018).

[52] T. O. Wehling, E. Şaşıŏlu, C. Friedrich, A. I. Lichtenstein, M. I. Katsnelson, and S. Blügel, Phys. Rev. Lett. 106, 236805 (2011).

[53] D. Jacob, K. Haule, and G. Kotliar, Phys. Rev. B 82, 195115 (2010).
[54] A. Valli, A. Amaricci, V. Brosco, and M. Capone, Nano Lett. 18, 2158 (2018).

[55] K. Haule, C.-H. Yee, and K. Kim, Phys. Rev. B 81, 195107 (2010).

[56] Y. Meir and N. S. Wingreen, Phys. Rev. Lett. 68, 2512 (1992).

[57] D. Jacob, J. Phys.: Condens. Matter 30, 354003 (2018).

[58] J. C. Slater, Phys. Rev. 36, 57 (1930).

[59] For smaller $\Gamma$ we need increasingly fine frequency meshes to properly resolve the low-energy PP peaks, which leads to higher computational cost as well as to numerical instabilities in the solution of the OCA equations.

[60] A. H. Nevidomskyy and P. Coleman, Phys. Rev. Lett. 103, 147205 (2009).

[61] F. Lombardi, A. Lodi, J. Ma, J. Liu, M. Slota, A. Narita, W. K. Myers, K. Müllen, X. Feng, and L. Bogani, Science 366, 1107 (2019).

[62] A. Gaita-Ariño, F. Luis, S. Hill, and E. Coronado, Nat. Chem. 11, 301 (2019). 